\title{
Segmented Terahertz Electron Accelerator and Manipulator (STEAM)
}

\author{
Dongfang Zhang ${ }^{1,2^{*} \dagger}$, Arya Fallahi ${ }^{1 \dagger}$, Michael Hemmer ${ }^{1}$, Xiaojun $\mathrm{Wu}^{1 \ddagger}$, Moein Fakhari ${ }^{1,2}$, Yi \\ Hua $^{1}$, Huseyin Cankaya ${ }^{1}$, Anne-Laure Calendron ${ }^{1,2}$, Luis E. Zapata ${ }^{1}$, Nicholas H. Matlis ${ }^{1}$, and
} Franz X. Kärtner ${ }^{1,2,3}$

\author{
Affiliations: \\ ${ }^{1}$ Center for Free-Electron Laser Science, Deutsches Elektronen Synchrotron, Notkestrasse 85, \\ 22607 Hamburg, Germany. \\ ${ }^{2}$ Department of Physics and The Hamburg Centre for Ultrafast Imaging, University of Hamburg, \\ Luruper Chaussee 149, 22761 Hamburg, Germany. \\ ${ }^{3}$ Research Laboratory of Electronics, MIT, Cambridge, 02139 Massachusetts, USA. \\ *To whom correspondence should be addressed. E-mail: dongfang.zhang@cfel.de \\ $\dagger$ †qual contribution. \\ $\ddagger$ Present address: School of Electronic and Information Engineering, Beihang University, \\ 100083, Beijing, China
}

\begin{abstract}
Acceleration and manipulation of electron bunches underlie most electron and X-ray devices used for ultrafast imaging and spectroscopy. New terahertz-driven concepts offer ordersof-magnitude improvements in field strengths, field gradients, laser synchronization and compactness relative to conventional radio-frequency devices, enabling shorter electron bunches and higher resolution with less infrastructure while maintaining high charge capacities (pC), repetition rates $(\mathrm{kHz})$ and stability. We present a segmented terahertz electron accelerator and manipulator (STEAM) capable of performing multiple high-field operations on the 6D-phase-
\end{abstract}


space of ultrashort electron bunches. With this single device, powered by few-micro-Joule, single-cycle, $0.3 \mathrm{THz}$ pulses, we demonstrate record $\mathrm{THz}$-acceleration of $>30 \mathrm{keV}$, streaking with $<10$ fs resolution, focusing with $>2 \mathrm{kT} / \mathrm{m}$ strength, compression to $\sim 100 \mathrm{fs}$ as well as realtime switching between these modes of operation. The STEAM device demonstrates the feasibility of THz-based electron accelerators, manipulators and diagnostic tools enabling science beyond current resolution frontiers with transformative impact.

\section{Main}

Particle accelerator development over the last century has underpinned the study of fundamental forces and particles as well as structure and function of materials and its properties at ever higher spatial and temporal resolution. Until recently, microwaves in the radio-frequency (RF) regime (1-10 GHz) have been the conventional choice for powering accelerators due to the high degree of technical maturity of the sources, which have been used extensively across all areas of industry and science, from cell phones, microwave ovens and radar to linear accelerators ${ }^{1}$, bunch compressors $^{2,3}$ and high-resolution streak cameras ${ }^{4,5}$. The long driver wavelengths are ideal for accelerating electron bunches with up to nanocoulomb bunch charge, and as a result of many decades of development, it has become possible to generate ultrafast electron pulses with very high peak brightness and quality. However, RF-based accelerators require costly infrastructures of large size and power ${ }^{6}$, limiting the availability of this key scientific resource. They also suffer from inherent difficulties in synchronization with lasers ${ }^{7}$ which lead to timing drifts on the 100femtosecond scale between the electrons, microwave drivers and optical probes, limiting the achievable temporal resolution. Strong motivation thus exists for exploring alternative technologies that are compact, more accessible and adapted for pushing the resolution frontier especially where lower levels of charge in the few $\mathrm{pC}$ range or lower is sufficient. Novel 
accelerator concepts thus primarily focus on laser-based approaches that provide intrinsic synchronization, allow scaling to smaller accelerator structures and can generate substantially stronger fields for acceleration and beam manipulation. These include dielectric laser accelerators $(\mathrm{DLAs})^{8,9}$, laser-plasma accelerators (LPAs) $)^{10-14}$ and laser-based THz-driven accelerators $^{15-17}$, each with different advantages. A consequence of downscaling in size is that less charge can be supported and creation of reliable structures can become more difficult. LPAs, for example, which boast extremely high acceleration gradients on the order of $100 \mathrm{GV} / \mathrm{m}$, generate acceleration structures dynamically and therefore suffer from instabilities and difficulties in controlling injection. DLAs, which employ micron-scale structures, require extreme tolerances on alignment and control, and are limited to bunch charges in the subfemtocoulomb range. THz-based accelerators, however, exist at an intermediate, millimeter scale that allows traditional fabrication techniques and supports moderate charge while still benefiting from compactness, low cost and strong driving fields. This balance makes THz-based acceleration an extremely promising technology for future devices.

So far, the development of THz-based accelerators has been limited by the lack of sufficiently energetic $\mathrm{THz}$ sources, but recent progress in efficient laser-based methods ${ }^{18-20}$ has enabled generation of high-power, GV/m THz fields, opening new possibilities and spurring interest in THz-accelerator-related technologies. Proof-of-principle demonstrations include electron emission $^{21,22}$ and acceleration ${ }^{15,16,23-27}$ as well as compression and streaking ${ }^{28,29}$. These experiments, although limited in charge, beam quality, energy gain and energy spread, have set the stage for development of practical, compact THz-based devices which can support sufficient charge and field gradients to realistically be used to boost performance of existing accelerators or as components of future compact accelerators and X-ray sources. In this letter, we demonstrate 
the first such device based on a layered, transversely pumped, waveguide structure. This segmented $\mathrm{THz}$ electron accelerator and manipulator (STEAM) device can dynamically switch between accelerating, streaking, focusing and compressing modes, can support multiple picocoulombs of charge and features intrinsic synchronization. Using only a few microjoules of single-cycle $\mathrm{THz}$ radiation, we demonstrate over $70 \mathrm{MV} / \mathrm{m}$ peak acceleration fields, $2 \mathrm{kT} / \mathrm{m}$ focusing gradients (which are an order of magnitude beyond current electromagnetic lenses and comparable to active plasma lenses), the highest reported $\mathrm{THz}$ streaking gradient of $140 \mu \mathrm{rad} / \mathrm{fs}$ (making it well-suited for characterization of ultrafast electron diffractometer bunches down to $10 \mathrm{fs)}$ as well as compression to $\sim 100 \mathrm{fs}$. All these demonstrations are strongly benefitting from very small temporal jitter achieved through laser-driven $\mathrm{THz}$ sources (see supplementary material). By increasing THz pulse energies to state-of-the-art millijoule levels ${ }^{20}$, it is expected that acceleration gradients approaching $1 \mathrm{GV} / \mathrm{m}$ can be achieved and sustained. Such gradients surpass those possible in RF accelerators by an order of magnitude and enable major improvements in electron bunch qualities such as emittance and bunch length. The picosecond duration of the $\mathrm{THz}$ pulses is an essential ingredient for reaching the $\mathrm{GV} / \mathrm{m}$ regime, since experiments have shown that maximum acceleration gradients, which are limited by fieldinduced breakdown, scale with the sixth power of the field duration ${ }^{30-33}$. Demonstration of the THz-driven STEAM device thus establishes a new compact, strong-field and extremely-highgradient accelerator technology.

\section{Concept and implementation}

The experimental setup (Fig. 1a) consisted of a $55 \mathrm{keV}$ photo-triggered DC gun, a THz-powered STEAM device for electron acceleration or manipulation and a diagnostic section which included a second STEAM device used as a streak camera, all of which were driven by the same 
IR laser source. Ultraviolet pulses for photoemission were generated by two successive stages of second harmonic generation, while single-cycle $\mathrm{THz}$ pulses were generated by difference frequency generation. THz from two independent setups was coupled into the STEAM device (Fig. 1) transversely to the electron motion by two horn structures which focused the counterpropagating $\mathrm{THz}$ fields beyond the diffraction limit into the interaction zone. The electrons experience both the electric and magnetic fields of the $\mathrm{THz}$ pulses according to the Lorentz force law $\vec{F}=q(\vec{E}+\vec{v} \times \vec{B})$, where $-q$ is the electron charge, $\vec{E}$ is the electric field, oriented parallel to the electron velocity $\vec{v}$, and $\vec{B}$ is the magnetic field, oriented vertically in the lab frame. The electric field is thus responsible for acceleration and deceleration, while the magnetic field induces transverse deflections.

Efficient interaction of the electrons with the fields was accomplished by means of segmentation which divided the interaction volume into multiple layers, each isolated from the others by thin metal sheets (Fig. 1). Dielectric slabs of varying length were inserted into each layer to delay the arrival time of the $\mathrm{THz}$ waveform to coincide with the arrival of the electrons, effectively phasematching the interaction. Due to the transverse geometry, the degree of dephasing experienced in each layer was determined by the traversal time of the electrons, which was dependent on the electron speed and the layer thickness. A reduction in dephasing can thus be accomplished by reducing the layer thickness and increasing the number of layers, at the cost of increased complexity. The ability to tune the thickness and delay of each layer independently is a key design feature of the STEAM device that enables acceleration of sub-relativistic electrons for which the speed changes significantly during the interaction (e.g, from $0.43 c$ to $0.51 c$ for our maximum acceleration case). 
The use of two counter-propagating drive pulses enabled two key modes of operation which are specified with respect to the interaction point, i.e., the center of the interaction region of each layer:

- an "electric" mode, used for acceleration, compression \& focusing, in which the pulses were timed to produce electric superposition and magnetic cancellation of the transverse fields at the interaction point;

- a "magnetic" mode, used for deflection and streaking, where the magnetic fields superposed and the electric fields cancelled.

The function of the device was thus selected by tuning the relative delay of the two $\mathrm{THz}$ pulses and the electrons, all of which were controlled by means of motorized stages acting on the respective infrared pump beams. In focusing and streaking modes the electron beams were sent directly to a microchannel plate (MCP) detector. For acceleration measurements, an electromagnetic dipole was used to induce energy-dependent deflections in the vertical plane, so that both deflection and energy change could be measured simultaneously. To measure the compression, a second STEAM device in streaking mode was added downstream of the first to induce time-dependent deflections in the horizontal plane. The breakdown threshold of the device is determined by field emission from the metallic parts. Owing to the 4-5 orders of magnitude shorter field-exposure times of single-cycle $\mathrm{THz}$ pulses as compared with $\mathrm{RF}$ excitations, the previous studies suggest that a factor of 3-10 higher breakdown threshold for pulsed $\mathrm{THz}$ driven devices can be expected ${ }^{26,31}$. The remainder of this paper gives a detailed description of the results obtained for acceleration, compression, focusing, deflection and streaking for this STEAM device. 


\section{Electric Mode: acceleration, compression \& focusing}

In the electric mode, the relative timing of the $\mathrm{THz}$ pulses was adjusted so that the electric fields ( $E$-fields) constructively interfered at the interaction point. In this configuration, the magnetic fields ( $B$-fields) were $180^{\circ}$ out of phase with each other and thus cancelled, minimizing unwanted deflections. The acceleration was sensitive to the $\mathrm{THz}$ phase at the interaction. Figure 2 shows energy and deflection diagrams which were obtained by recording the vertical and horizontal projections (respectively) of the electron-beam distribution on the MCP as a function of the electron- $\mathrm{THz}$ delay. Although the $\mathrm{THz}$ pulses injected into the device were nearly single cycle, several cycles of acceleration and deceleration were observed, due to dispersion induced by the horn couplers.

Maximum acceleration and deceleration occurred at the electron injection points (Fig. 2a) where the deflection was minimized (Fig. 2c) and the beam spatial distribution was also preserved (Fig. 2e left \& right beams). The peak field is calculated to have reached $\sim 70 \mathrm{MV} / \mathrm{m}$ with the Yb:YLF laser, based on comparisons of the measured $\mathrm{THz}$ energy transmitted through the device and the electron energy gain with simulation (described below). The energy gain scaled linearly with the applied field (Fig. 3b) and reached a record of more than $30 \mathrm{keV}$ (5 times larger than previous studies $^{15}$ ) for a bunch charge of $\sim 5 \mathrm{fC}$. In contrast to previous results showing simultaneous acceleration and deceleration, the energy spectrum can be seen to move cleanly to a higher energy (Fig. 3a), indicating that injected bunches were shorter than half the driver period. In fact, the bunches were measured (by the STEAM device in streaking mode) to have a duration of 670 fs, and thus occupied about $20 \%$ of the 3.33 ps period accelerating field. The increase in energy spread is attributed in part to the variation of the $E$-field over the bunch temporal profile. Although bunches with charge up to $20 \mathrm{fC}$ were coupled into the device, space-charge effects 
and the long travel distances from the DC gun lead to longer bunch duration and larger energy spread. For demonstrating THz-driven acceleration, the charge was limited to 1-5 fC during this experiment. Use of a THz-based re-buncher before the accelerator is thus anticipated for future experiments to reduce energy spread.

The performance of the device was simulated using a finite-element based code ${ }^{34}$. Figure $3 \mathrm{c}$ shows snapshots of the electrons traversing the device and staying in phase with the field (full simulation results are presented in movie $\mathrm{S} 1$ ). Figure $3 \mathrm{~d}$ shows the electron energy as a function of distance. The energy gain can be seen to occur in three uneven steps corresponding to the three layers. The unevenness and the presence of deceleration at some points are evidence of dephasing due to the fact that the structure was designed for higher $\mathrm{THz}$ energies (see supplementary material). Simulations predict that $\mathrm{MeV}$ electron beams with up to $10 \mathrm{pC}$ of charge are achievable by increasing the number of layers and extending $\mathrm{THz}$ pulse energies to the millijoule level ${ }^{24}$ which is within the reach of current $\mathrm{THz}$-generation methods ${ }^{20}$.

At timings off from the optimum acceleration, the electrons experienced strong temporal gradients of the $E$-field resulting in large energy spreads (Fig. 2a). At the zero-crossing of the field, the gradient is maximized and the electrons see symmetric acceleration and deceleration but no net energy gain. In this mode, the $E$-field imparts a temporally-varying energy or "chirp" resulting in a velocity gradient that causes either compression or stretching (depending on the sign of the gradient) of the electron bunch as it propagates ${ }^{35}$. This technique, known as "velocity bunching" is an ideal application of $\mathrm{THz}$ technology, since the sub-mm-scale gradients allow bunch compression down to the femtosecond range. To test this concept, the applied THz energy was varied and a second STEAM device ("streaker") acting as a streak camera (described in the 
next section) was added to measure the bunch temporal profile at a point $200 \mathrm{~mm}$ downstream of the first device ("buncher").

Figure 4a shows the electron bunch temporal profiles measured at the streaker for various field strengths applied to the buncher. The initial decrease in bunch duration with increasing field confirms that the electrons arrive at the buncher with a space-charge-induced energy chirp. A minimum duration of $\sim 100$ fs FWHM was achieved after which the duration increases again (Fig. 4b), implying that for high fields the electrons temporally focus before the streaker and are over compressed by the time they are measured. The minimum bunch duration can thus be reduced by using stronger fields and a shorter propagation distance as shown by the simulation in Supplementary Fig. S7. As observed on the MCP-detector (Fig. 4b inset), a good e-beam profile is maintained at the optimal compressed condition. Figure $4 \mathrm{c}$ shows the evolution of the bunch duration with distance simulated for the minimum bunch duration case. The phase-space distributions in insets show the reversal of the velocity correlation by the buncher and the eventual compression at the streaker location.

By placing the electrons at the zero crossing in the electric mode (corresponding to the maximum $B$-field rotating around the interaction region), the STEAM device can also operate as a focusing or defocusing element, as can be seen by the horizontal spreading of the beam profile in Figure 2c. Due to the strong THz field that leads to over-focusing at the fixed MCP position, both focusing and defocusing schemes are observed here as an increase of the beam size. This focusing effect is a consequence of the well-known Panofsky-Wenzel Theorem ${ }^{36}$, which uses Gauss's law to show that longitudinal compressing and decompressing fields must be accompanied by transverse defocusing and focusing fields, respectively. The $B$-field always cancels at the interaction point, while still has time varying transverse magnetic field distribution 
in the antinode region that contributes to the defocusing and focusing (illustrated in Fig. 5c,d). The focusing was tested by monitoring the beam spatial profile at the MCP for varying $\mathrm{THz}$ pulse energies. Figure 5a shows the results for the focusing configuration, which corresponded to the longitudinal decompression condition. At best, the electron beam diameter was reduced by $2 \times$ compared with its input value. For higher field strengths, however, the device focal length became shorter than the $180 \mathrm{~mm}$ distance to the MCP, causing the measured beam size to increase again. Similarly to photon beams, a focusing optics with higher focusing power results in a smaller beam at focus, provided that the input beam size is constant (see supplementary material). The defocusing configuration is obtained by shifting the electron timing to the longitudinal compression condition, which occurs at an adjacent zero crossing of opposite sign. In this case, the electron beam diameter increases monotonically with the THz field (Fig. 5b), as expected. For both cases, the focusing performance is significantly beyond what is offered by conventional electrostatic $^{37}$ and proposed dielectric focusing structures ${ }^{38}$ and is comparable to those of plasma lenses ${ }^{39}$. Peak focusing gradients of $>2 \mathrm{kT} / \mathrm{m}$ were calculated based on $\sim 2 \times 6 \mu \mathrm{J}$ of coupled THz energy. A small (less than a factor of 2) asymmetry is noticeable for the focusing strengths in the horizontal and vertical planes. This asymmetry is due to the asymmetry of the interaction region which leads to stronger gradients in the vertical direction (Fig. 5c,d).

\section{Magnetic Mode: Deflection \& streaking}

In the magnetic mode, the relative timing of the $\mathrm{THz}$ fields is different from that of the electric mode by a half period, resulting in reinforcement of the magnetic and cancellation of the electric fields at the interaction region. In this configuration, electron acceleration is minimized (Fig. 2b), and the $B$-field dominates the interaction causing a transverse deflection of the electron beam that depends on the THz phase at the interaction (Fig. 2d). When electrons sweep the positive 
cycle of the $B$-field, the deflection is maximized and the beam profile is also best preserved. In this mode, electron beams can be precisely steered (Fig. 2e top \& bottom beams) by varying the $\mathrm{THz}$ pulse energy. Here, we achieved continuous control of the beam angle over a range of 70 mrad which was limited by the aperture of the device (Supplementary Fig. S6). Increasing the aperture enables greater range at the cost of a weaker deflection field, since the field confinement is affected.

Electrons sweeping the zero crossing cycle of the $\mathrm{THz} B$-field, on the other hand, experience a strong deflection as a function of delay time enabling the measurement of the temporal bunch profiles of very short bunches by mapping (or "streaking") them onto the spatial dimension of a detector. To test this concept, a first STEAM device was used in compression mode (as described above) to provide electrons of varying bunch durations at a second, downstream STEAM device which analyzed the temporal profiles by streaking. Figure 6a shows raw images of a temporally-long electron beam with the THz streaking field switched on and off. Streaking "deflectograms," generated by plotting a lineout of the spatial charge distribution along the streaking dimension as a function of delay relative to the $\mathrm{THz}$ field, are shown in Fig. $6 \mathrm{~b}$ for compressed and uncompressed electron bunches. The degree of streaking, indicated by the vertical extent of the deflectogram, depends clearly on the bunch duration and on the phase of the $\mathrm{THz}$ field, as expected. For a THz energy of $\sim 2 \times 6 \mu \mathrm{J}$ coupled into the device, a maximum deflection rate of $>140 \mu \mathrm{rad} / \mathrm{fs}$ was achieved (Supplementary Fig. S7), corresponding to a temporal resolution below 10 fs. The resolution was limited here by the $350 \mu \mathrm{m}$ size of the unstreaked beam. These results represent a new record in THz-based streaking gradient as well as the first use of $\mathrm{THz} B$-fields for deflection and streaking. 


\section{Conclusions and outlook}

We have demonstrated a novel segmented $\mathrm{THz}$ electron accelerator and manipulator setting new records in $\mathrm{THz}$ acceleration, streaking and focusing with a very compact device. The segmented structure makes it possible to phase-match the electron- $\mathrm{THz}$ interaction for non-relativistic beams, making it ideal for use as a high-gradient photogun ${ }^{24}$. The independent control over the counter-propagating $\mathrm{THz}$ pulse timing gives the STEAM device the ability to switch dynamically between acceleration, compression, focusing, deflection and streaking modes. As was theoretically shown in ref. 24 , the use of $\mathrm{THz}$ pulses also brings other advantages, including negligible heat loads, high repetition rates and compactness while still supporting substantial charge in the $\mathrm{pC}$ regime. Furthermore, the three-layer structure demonstrated in this study indicates the path forward towards relativistic electron energies by staging more layers for higher operation efficiency.

Using only $\sim 2 \times 6 \mu \mathrm{J}$ of $\mathrm{THz}$ energy the STEAM device has demonstrated peak acceleration gradients of $70 \mathrm{MV} / \mathrm{m}$, compression of a bunch from over $1 \mathrm{ps}$ to $100 \mathrm{fs}$, focusing strength of $\sim 2$ $\mathrm{kT} / \mathrm{m}$ and streaking gradients of $>140 \mu \mathrm{rad} / \mathrm{fs}$, leading to a temporal resolution below $10 \mathrm{fs}$. By scaling to millijoule-level $\mathrm{THz}$ energies which are already available in some $\mathrm{THz}$ wavelength ranges, the field strengths in the device can be increased by over an order of magnitude far exceeding those of conventional RF devices. The exceptional performance and compactness of this THz-based device makes it very attractive for pursuing electron sources, like ultrafast electron diffractometers, that operate in the few- and sub-fs range necessary for probing the fastest material dynamics ${ }^{40,41}$. In the pursuit of these sources, the demand is increasing for compact, high-gradient diagnostics and beam manipulation devices for novel and conventional accelerator platforms alike. In large-scale facilities, such as the European X-ray free-electron 
laser (XFEL), the Linac Coherent Light Source (LCLS) or the Swiss free-electron laser (SwissFEL), the STEAM devices can be used to add new, powerful and adaptable capabilities without major and therefore costly restructuring of the machine. More significant are the advantages in terms of cost and accessibility that come from using STEAM devices as the core components of an all-THz-powered compact, high-gradient accelerator with the ability to produce high-quality, controllable bunches of femtosecond or attosecond duration on a table top. The results presented here are the first step in demonstrating the feasibility of that vision.

\section{References:}

1. European X-Ray Free-Electron Laser Facility https://www.xfel.eu/index_eng.html

2. Gao, M. et al. Full characterization of RF compressed femtosecond electron pulses using ponderomotive scattering. Opt. Express 20, 12048-12058 (2012).

3. Maxson, J., et al. D. Direct Measurement of Sub-10 fs Relativistic Electron Beams with Ultralow Emittance. Phys. Rev. Lett. 118, 154802 (2017).

4. Floettmann, K. \& Paramonov, V.V., Beam dynamics in transverse deflecting rf structures, Phys. Rev. Accel. Beams 17, 024001 (2014).

5. Ischebeck, R., Prat, E., Thominet, V. \& Loch, C. O. Transverse profile imager for ultrabright electron beams, Phys. Rev. Accel. Beams 18, 082802 (2015).

6. Manz, S. et al. Mapping atomic motions with ultrabright electrons: towards fundamental limits in space-time resolution. Faraday Discuss. 177, 467-491 (2015).

7. Yang, H. et al.10-fs-level synchronization of photocathode laser with RF-oscillator for ultrafast electron and X-ray sources. Sci. Rep. 7, 39966 (2017). 
8. Peralta, E. A. et al. Demonstration of electron acceleration in a laser-driven dielectric microstructure. Nature 503, 91-94 (2013).

9. Breuer, J. \& Hommelhoff, P. Laser-Based Acceleration of Nonrelativistic Electrons at a Dielectric Structure. Phys. Rev. Lett. 111, 134803 (2013).

10. Malka, V. et al. Principles and applications of compact laser-plasma accelerators. Nature Physics 4, 447-453 (2008).

11. Leemans, W. \& Esarey, E. Laser-driven plasma-wave electron accelerators. Phys. Today 62, 44-49 (2009).

12. Guénot, D. et al. Relativistic electron beams driven by kHz single-cycle light pulses. Nature Photonics 11, 293-296 (2017).

13. Salehi, F. et al. MeV electron acceleration at $1 \mathrm{kHz}$ with $<10 \mathrm{~mJ}$ laser pulses. Opt. Lett. 42, 215-218 (2017).

14. He, Z-H. et al. High repetition-rate wakefield electron source generated by few-millijoule, 30 fs laser pulses on a density downramp. New J. Phys. 15, 053016 (2013).

15. Nanni, E. A. et al. Terahertz-driven linear electron acceleration. Nat. Commun. 6, 8486 (2015).

16. Walsh, D. A. et al. Demonstration of sub-luminal propagation of single-cycle terahertz pulses for particle acceleration. Nat. Commun. 8, 421 (2017).

17. Curry, E., Fabbri, S., Maxson, J., Musumeci, P. \& Gover, A. Meter-Scale Terahertz-Driven Acceleration of a Relativistic Beam. Preprint at https://arxiv.org/abs/1708.06385 (2017).

18. Hebling, J., Almasi, G., Kozma, I. Z. \& Kuhl, J. Velocity matching by pulse front tilting for large area THz-pulse generation. Opt. Express 10, 1161-1166 (2002). 
19. Huang, S.-W. et al. High conversion efficiency, high energy terahertz pulses by optical rectification in cryogenically cooled lithium niobate. Opt. Lett. 38, 796-798 (2013).

20. Fülöp, J. A. et al. Efficient generation of THz pulses with 0.4 mJ energy. Opt. Express 22 , 20155-20163 (2014).

21. Wimmer, L. et al. Terahertz control of nanotip photoemission. Nat. Phys. 10. 432-436 (2014).

22. Li, S. \& Jones, R. R. High-energy electron emission from metallic nano-tips driven by intense single-cycle terahertz pulses. Nat. Commun. 7, 13405 (2016).

23. Huang, R. W. et al. Toward a terahertz-driven electron gun. Sci. Rep. 5, 14899 (2015).

24. Fallahi, A., Fakhari, M., Yahaghi, A., Arrieta, M. \& Kärtner, F. X. Short Electron Bunch Generation Using Single-Cycle Ultrafast Electron Guns. Phys. Rev. Accel. Beams 19, 081302 (2016).

25. Hebling, J. et al. Optical manipulation of relativistic electron beams using THz pulses. Preprint at http://arxiv.org/abs/1109.6852 (2011).

26. Huang, W. R. et al. Terahertz-driven, all-optical electron gun. Optica 3, 1209-1212 (2016).

27. Fakhari, M., Fallahi, A. \& Kärtner, F. X. THz cavities and injectors for compact electron acceleration using laser-driven THz sources. Phys. Rev. Accel. Beams 20, 041302 (2017). 28. Fabiańska, J., Kassier, G. \& Feurer, T. Split ring resonator based THz-driven electron streak camera featuring femtosecond resolution. Sci. Rep. 4, 5645 (2014).

29. Kealhofer, C. et al. All-optical control and metrology of electron pulses. Science 352, 429$433(2016)$

30. Döbert, S. et al. High Gradient Performance of NLC/GLC X-Band Accelerating Structures. in Proceedings of Particle Accelerator Conference, 372-374 (IEEE, 2005). 
31. Massimo, D. F. et al. Experimental measurements of rf breakdowns and deflecting gradients in mm-wave metallic accelerating structures. Phys. Rev. Accel. Beams 19, 051302 (2016). 32. Wu, X. et al. High-gradient breakdown studies of an X-band Compact Linear Collider prototype structure. Phys. Rev. Accel. Beams 20, 052001 (2017).

33. Kilpatrick, W. Criterion for vacuum sparking designed to include both $\mathrm{rf}$ and dc. Rev. Sci. Instrum. 28, 824-826 (1957).

34. Fallahi, A. \& Kärtner, F. X. Field-based DGTD/PIC technique for general and stable simulation of interaction between light and electron bunches. J. Phys. B 47, 234015 (2014).

35. van Oudheusden, T., de Jong, E.F., van der Ceer, S.B., Op't Root, W.P.E.M. \& Juiten, O.J. Electron source concept for single-shot sub-100 fs electron diffraction in the $100 \mathrm{keV}$ range. $J$. Appl. Phys. 102, 093501 (2007).

36. Panofsky, W. K. H. \& Wenzel, W. A. Some considerations concerning the transverse deflection of charged particles in radio - frequency fields. Rev. Sci. Instrum. 27, 967 (1956).

37. Cesar, D. et al. Demonstration of Single-Shot Picosecond Time-Resolved MeV Electron Imaging Using a Compact Permanent Magnet Quadrupole Based Lens. Phys. Rev. Lett. 117, 024801 (2016).

38. Wootton, K. P. et al. Dielectric laser acceleration and focusing using short-pulse lasers with an arbitrary laser phase distribution. AIP Conf. Proc. 1812, 060001 (2017).

39. van Tilborg, J. et al. Active Plasma Lensing for Relativistic Laser-Plasma-Accelerated Electron Beams. Phys. Rev. Lett. 115, 184802 (2015).

40. Gliserin, A., Walbran, M., Krausz, F. \& Baum, P. Sub-phonon-period compression of electron pulses for atomic diffraction. Nat. Commun. 6, 8723 (2015). 
41. Sciaini, G. \& Miller, R. J. D. Femtosecond electron diffraction: heralding the era of atomically resolved dynamics. Rep. Prog. Phys. 74, 096101 (2001). 


\section{Figures}

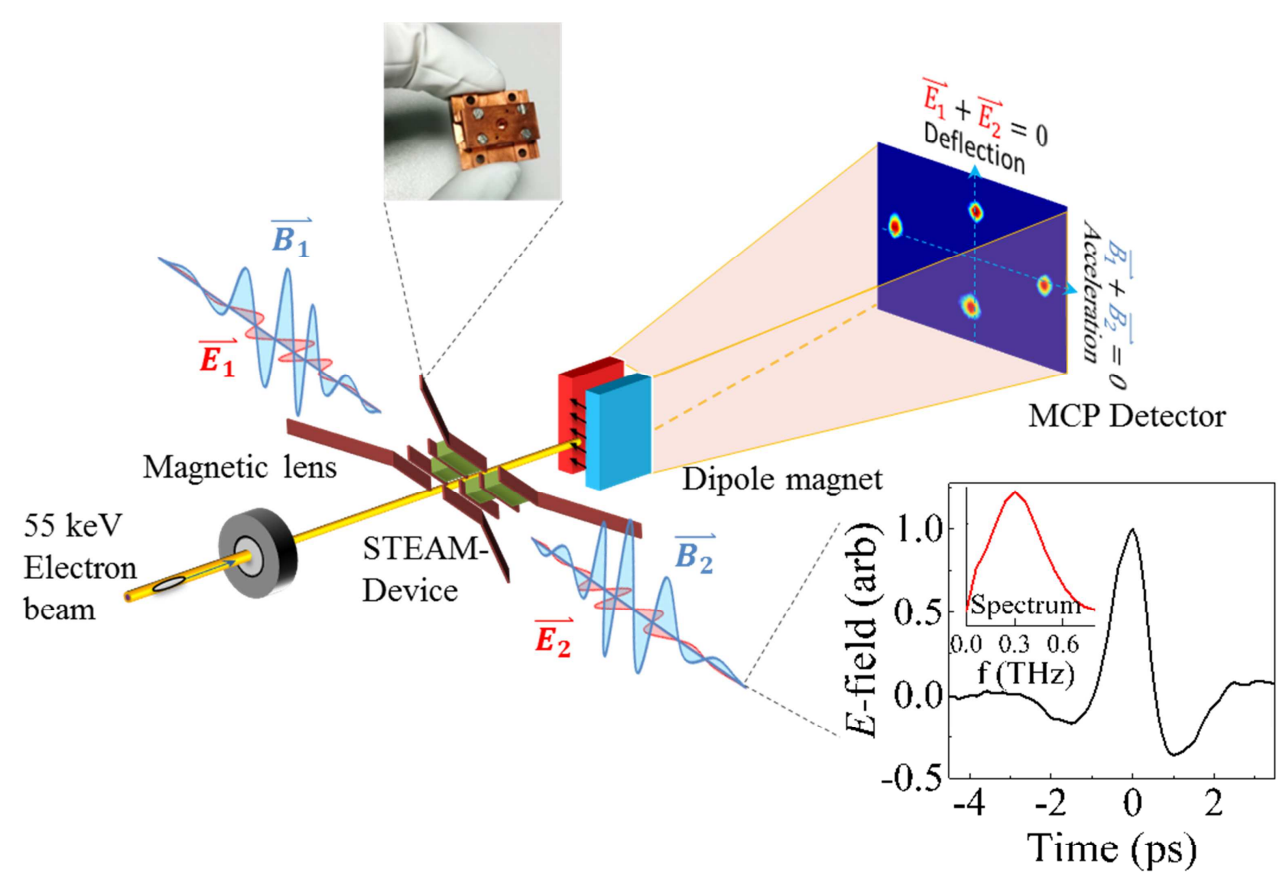

Fig. 1. Experimental setup. A fraction of the infrared optical beam is converted to $257 \mathrm{~nm}$ through fourth harmonic generation. The $257 \mathrm{~nm}$ laser pulse is directed onto a gold photocathode generating electron pulses, which are accelerated to $55 \mathrm{keV}$ by a DC electric field. This laser also drives two optical-rectification stages, each generating single-cycle terahertz pulses with energy up to $30 \mu \mathrm{J}$. The two counter-propagating $\mathrm{THz}$ beams interact with the electron beam inside the segmented structure. Subsequently, the electron beam is detected by the camera. Top left inset: photograph of the STEAM device. Bottom right inset: the time-domain waveform of the terahertz pulse measured by electro-optic sampling and its corresponding frequency (f)-domain spectrum. 


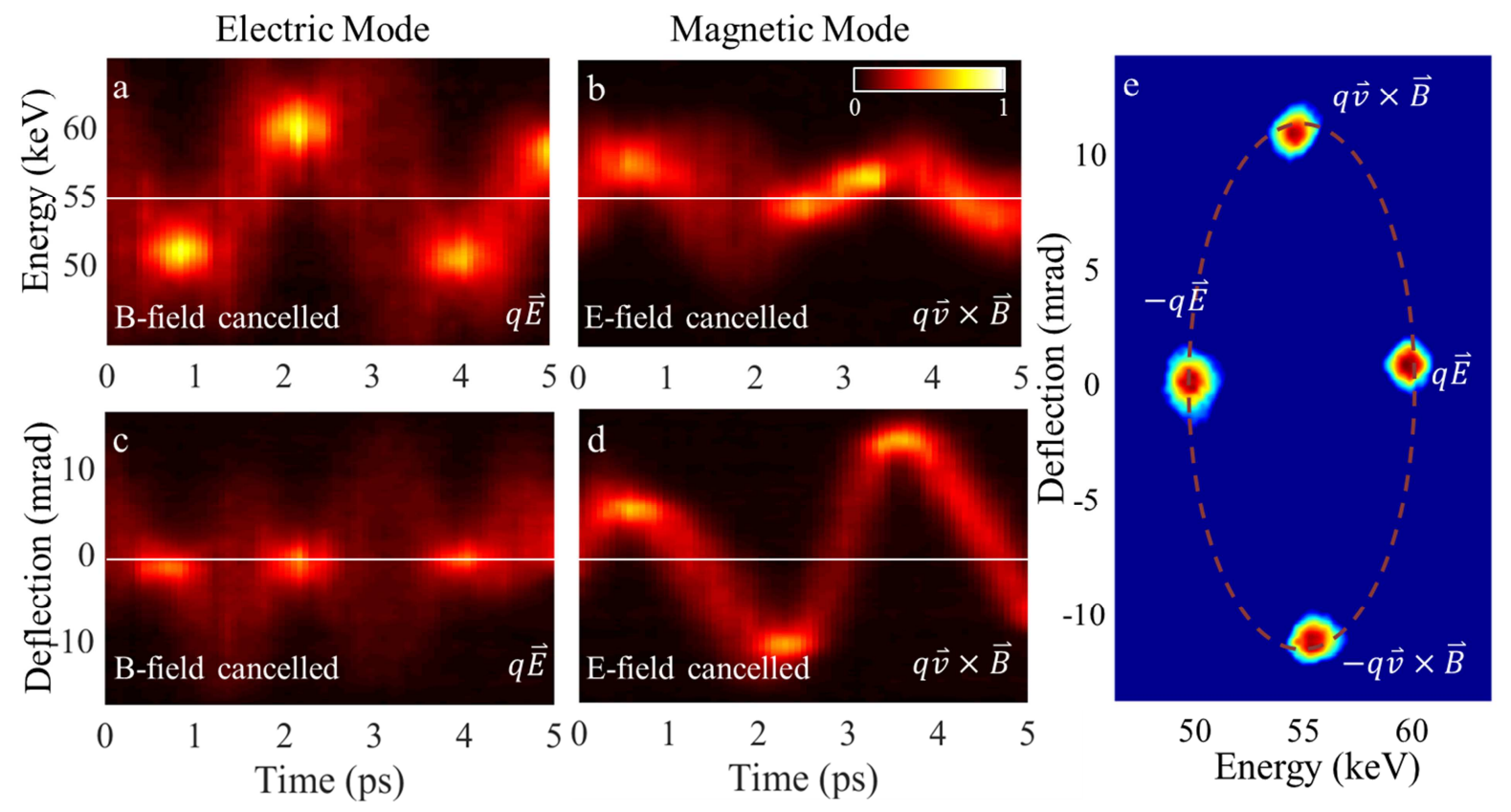

Fig. 2 Concept and implementation. a, Measured energy modulation of e-pulse as a function of electron-THz delay for constructive interference of the $E$-fields entering the device and cancellation of $B$-fields. b, Corresponding beam deflection measured for constructively interfering of the $B$-fields, i.e. $E$-field cancellation scenario. (c) and (d): Time-dependent deflection diagram measured by varying the electron-THz delay in the $B$-field and $E$-field cancellation scenario, respectively. e, Measured shape of e-beam on MCP detector for maximum acceleration, deceleration and right and left deflection points plotted in one image. Intensity was normalized and image contrast was tuned in order to show the relative positions more clearly. This demonstration was performed using the $\mathrm{Yb}: \mathrm{KYW}$ laser with $\sim 2 \times 0.5 \mu \mathrm{J}$ of THz radiation coupled into the device and a bunch charge of $\sim 1 \mathrm{fC}$. 
a

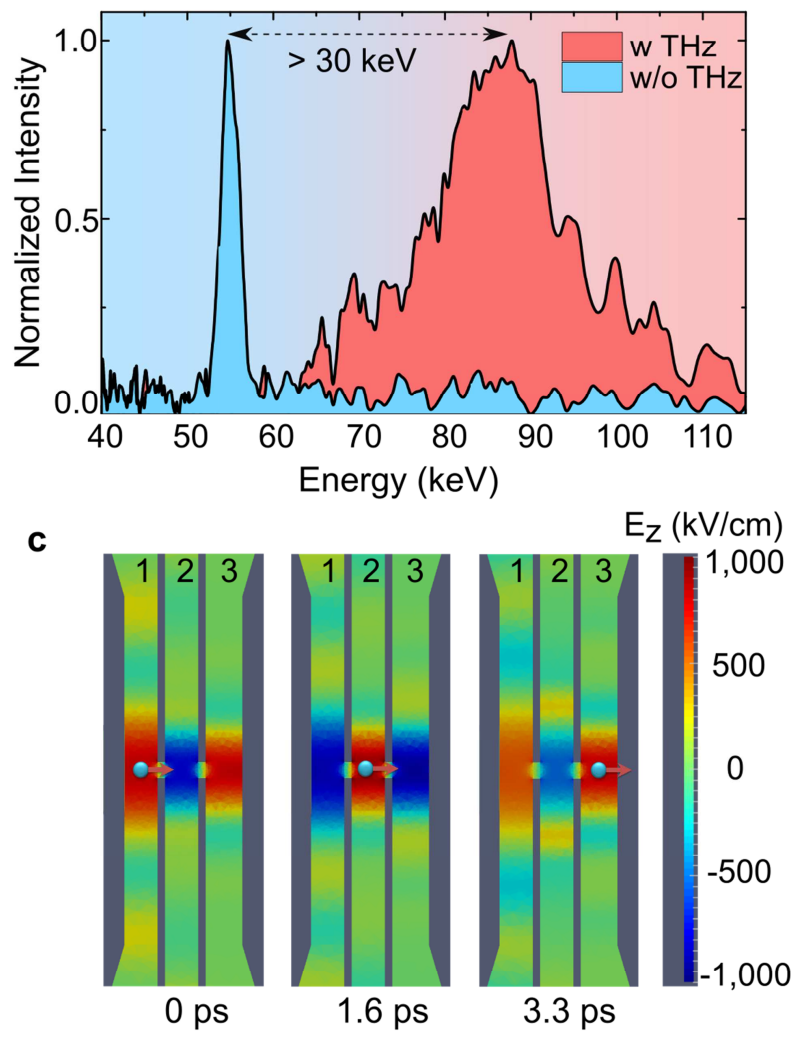

b

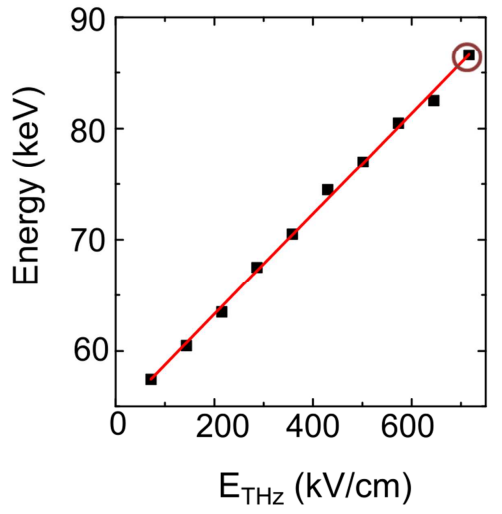

d

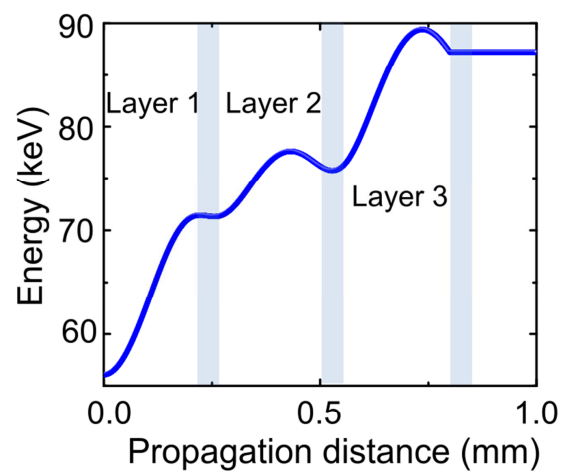

Fig. 3 Terahertz acceleration. a, Measured electron energy spectra for input beam (blue curve) and accelerated beam (red curve) after the STEAM-device that shows an energy gain of more than $30 \mathrm{keV}$. An increased energy spread is observed due to the long length of the initial electron bunch, as well as the slippage between the THz pulse and the electron bunch. b, Relative energy versus input terahertz field strength with red circle indicating the energy spectra plotted in (a)The linear relationship supports a direct, field-driven interaction. c, Temporal evolution of the electric field inside each layer with the red arrow indicating the electron propagating. d, Calculated acceleration along the electron propagation direction with $\sim 2 \times 6 \mu \mathrm{J} \mathrm{THz}$ and beam diameter of 3 $\mathrm{mm}$. This illustration was performed for the experiment using the Yb:YLF laser with $\sim 2 \times 6 \mu \mathrm{J}$ $\mathrm{THz}$ radiation coupled into the device and a bunch charge of $\sim 5 \mathrm{fC}$. 
a

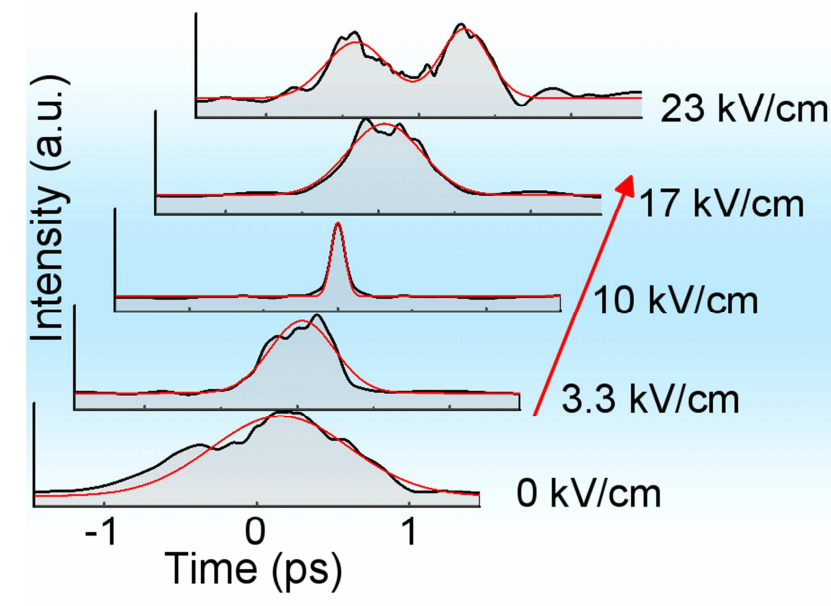

b

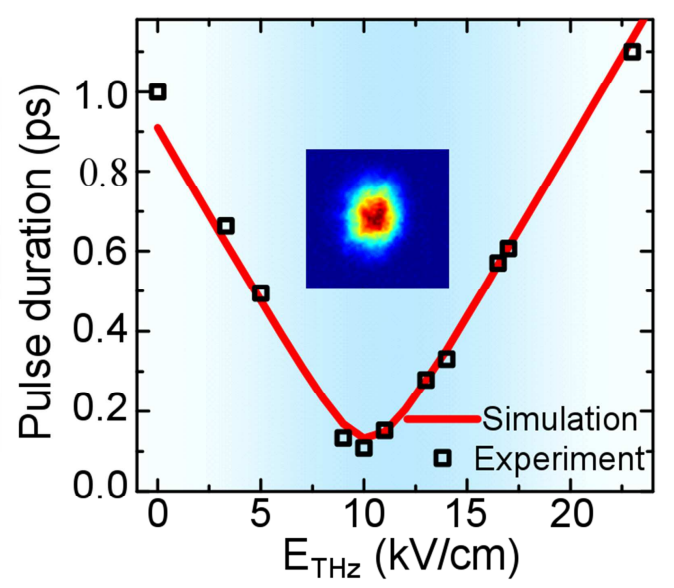

C

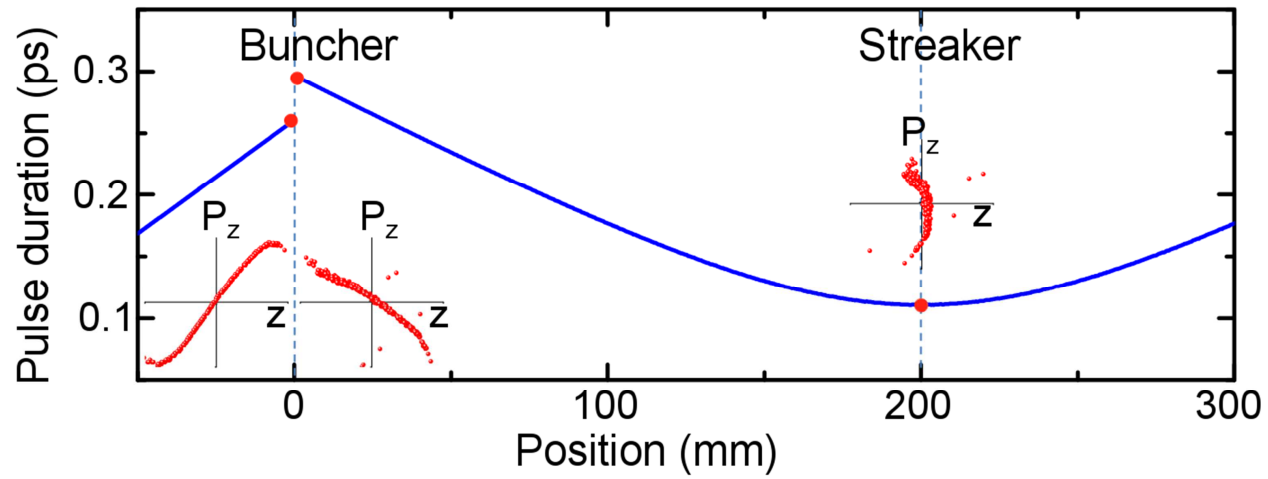

Fig. 4 Terahertz-driven electron pulse compression. a, Measured temporal profiles of the electron pulses as the THz field in the buncher is increased (red arrow). The red lines represent Gaussian fits. b, Black squares: measured electron bunch FWHM duration versus incident $\mathrm{THz}$ field strength. Red line: corresponding simulation results. Inset: the electron beam spatial profile on the detector at the optimal compressed condition. c, Simulated bunch length vs position. Inset: longitudinal phase-space distribution before the rebunching cavity, after the rebunching cavity and maximally compressed position marked with red dots respectively. This demonstration was performed with the $\mathrm{Yb}$ :KYW laser using one STEAM-device as a rebunching cavity and one as electron streak camera with a bunch charge of $\sim 1 \mathrm{fC}$. 


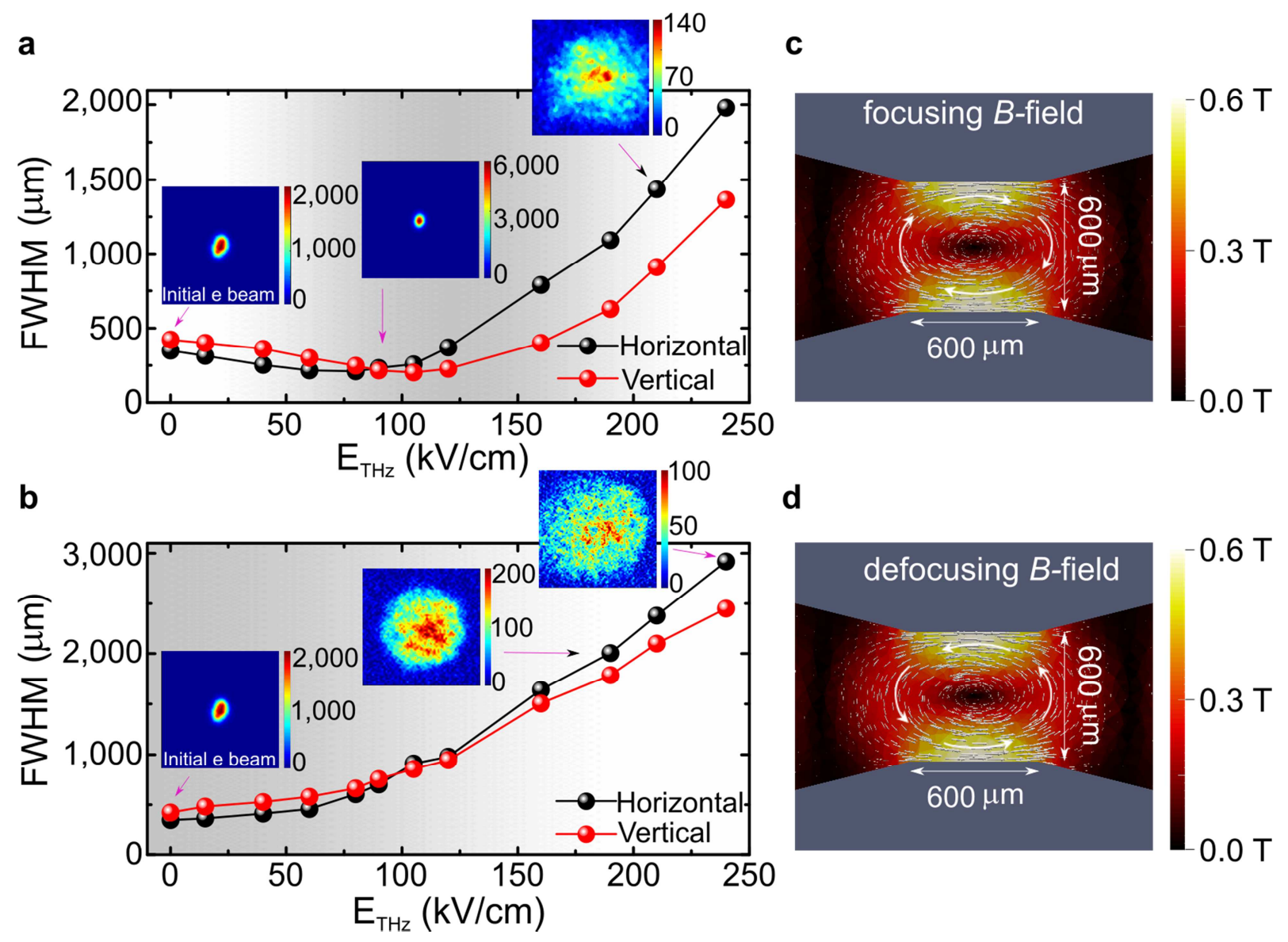

Fig. 5 Terahertz-lens for electron pulse focusing/defocusing. a,b, Measured transverse electron beam size at the MCP as a function of terahertz field for electron pulse focusing (a) and defocusing (b). This demonstration was performed with the Yb:KYW laser and a bunch charge of $\sim 1$ fC. Insets: spatial profiles of initial and focused and defocused electron beams on the MCP-detector. Legend shows the number of counts on the detector. c,d, Computed spatial field distributions of the focusing and defocusing fields, respectively. Simulation is performed with $\sim 2 \times 6 \mu \mathrm{J} \mathrm{THz}$ and beam diameter of $3 \mathrm{~mm}$. 


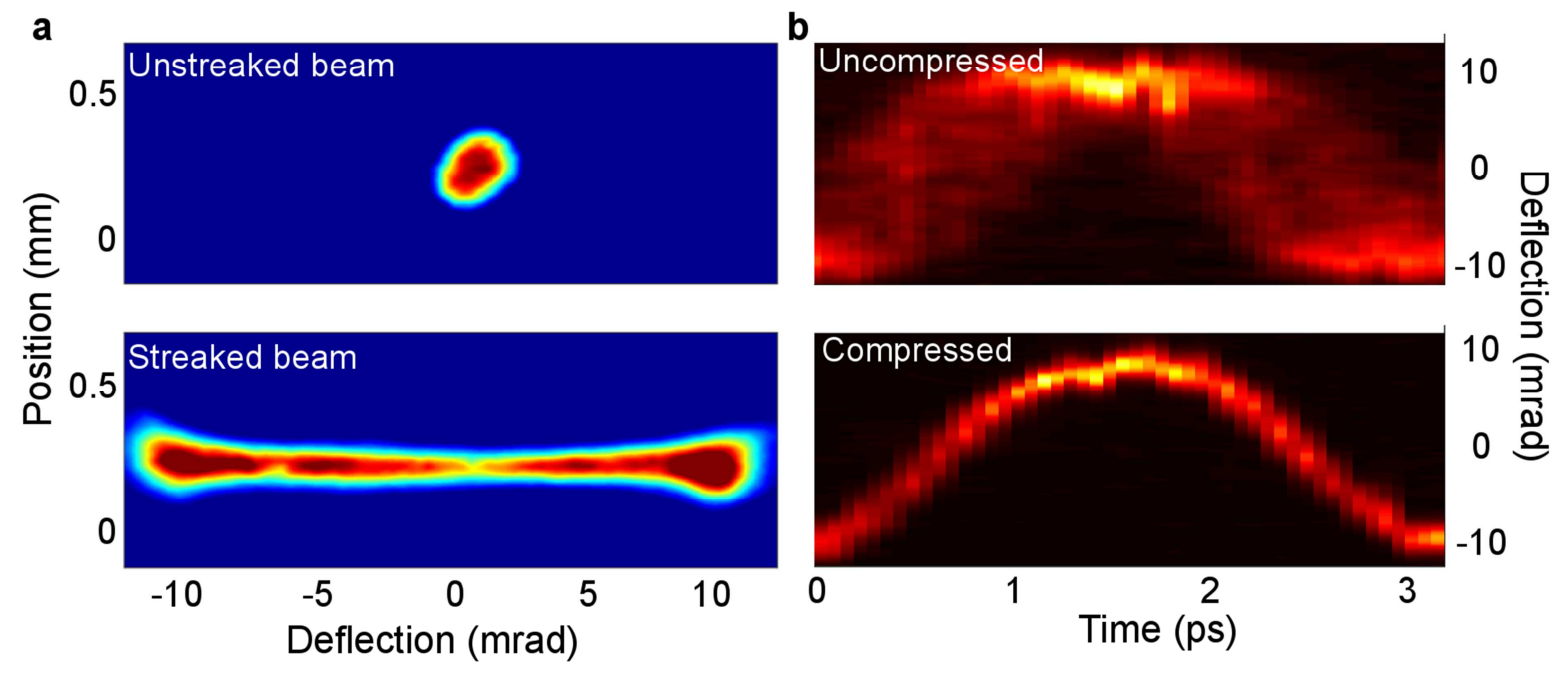

Fig. 6. Terahertz streak camera. a, Measured images of the electron beam on the MCPdetector with and without the terahertz deflection field. b, Time-dependent deflection diagram measured by varying the delay between the arrival time of electron bunch and the deflecting terahertz pulse for initially compressed and uncompressed electron bunches. This demonstration was performed with the $\mathrm{Yb}: \mathrm{KYW}$ laser and a bunch charge of $\sim 1 \mathrm{fC}$, except for the maximum streaked beam (a), where a bunch charge of $\sim 10 \mathrm{fC}$ is used in order to get a long pulse for demonstration. Deflectograms using the Yb:YLF laser can be found in the supplementary material showing more than 70 mrad deflection. 


\section{Methods}

Laser systems. Experiments were performed both with a 550fs, $4 \mathrm{~mJ}, 1,030 \mathrm{~nm} \mathrm{Yb:KYW} \mathrm{laser}$ operating at $1 \mathrm{kHz}$, to demonstrate high repetition rates, as well as with a $1.1 \mathrm{ps}, 40 \mathrm{~mJ}$, 1,020 nm Yb:YLF laser operating at $10 \mathrm{~Hz}$, in order to demonstrate high peak accelerations. Using these systems, THz pulses with a center frequency of $0.3 \mathrm{THz}$ were generated (Fig. 1 inset) by the well-established tilted pulse-front method ${ }^{15}$ from a $\mathrm{LiNbO}_{3}$ crystal, resulting in $2 \times 2$ $\mu \mathrm{J}$ pulses and $2 \times 30 \mu \mathrm{J}$ from the $\mathrm{Yb}: \mathrm{KYW}$ and $\mathrm{Yb}$ :YLF laser systems, respectively (supplementary material). Substantial losses due to transport and coupling of the THz beam, however, resulted in pulse energies at the interaction region of only $2 \times 0.5 \mu \mathrm{J}$ and $2 \times 6 \mu \mathrm{J}$ for the $\mathrm{Yb}: \mathrm{KYW}$ and Yb:YLF, respectively. The beam diameter at focus was measured to be $3 \mathrm{~mm}$. This diameter was also used for the corresponding simulations.

STEAM device dimensions. The STEAM device was designed with three layers of thickness $h=\{0.225,0.225,0.250\} \mathrm{mm}$ and with dielectric slabs in the second and third layers made of fused silica $\left(\epsilon_{\mathrm{r}}=4.41\right)$ and of length $L=\{0.42,0.84\} \mathrm{mm}$. The entrance and exit apertures were of diameter $120 \mu \mathrm{m}$. Further design parameters for the structure are shown in Supplementary Fig. S1.

Acknowledgments: We gratefully acknowledge helpful discussions with Chun Zhou, Wenqian Ronny Huang, Frederike Ahr, Wenchao Qiao, the expert technical support of Thomas Tilp, and Matthias Schust for fabrication of the STEAM-devices used in this work. Besides Deutsches Elektronen-Synchrotron (DESY) and the Helmholtz Association, this work has been supported by the European Research Council under the European Union's Seventh Framework Programme (FP7/2007-2013) through the Synergy Grant 'Frontiers 
in Attosecond X-ray Science: Imaging and Spectroscopy’ (AXSIS) (609920) and the excellence cluster "The Hamburg Center for Ultrafast Imaging - Structure, Dynamics and Control of Matter at the Atomic Scale" (CUI, DFG-EXC1074), the priority program 'Quantum Dynamics in Tailored Intense Fields' (QUTIF) (SPP1840 SOLSTICE) of the Deutsche Forschungsgemeinschaft and the accelerator on a chip program (ACHIP) funded by the Gordon and Betty Moore foundation (GBMF4744). The authors also thank Dr. T. Y. Fan and Dr. J. Zayhowski from MIT Lincoln Laboratory for initial work on the cryogenic Yb:YLF laser within the AXIS Program funded by the Defense Advanced Research Projects Agency (DARPA) and DARPA for the loan of the laser. Xiaojun Wu acknowledges support through Georg Forster Research Fellowship of the Alexander von Humboldt Foundation and Anne-Laure Calendron through a Helmholtz Postdoctoral Fellowship from the Helmholtz Association. Author contribution: F.X.K., D.Z., A.F. and N.H.M. conceived and coordinate the THz-driven electron acceleration and manipulation project. The structure was designed by A.F. and M.F. D.Z. designed the experimental setup and carried out the experiments. M.H., L.E.Z. and Y.H. built the Yb:YLF laser. A.-L.C. built the Yb:KYW laser with the help of H.C. X.W. and D.Z. built the THz setup. D.Z. built the UV generation and automated the setup. A.F. performed all simulations. A.-L.C., H.C., M.H., Y.H. and L.E.Z. maintained the laser systems and contributed with helpful discussions on the experiment. D.Z., A.F., N.H.M. and F.X.K. wrote the manuscript with revisions by all.

\section{Supplementary Information}

Supplementary Fig. S1-S9.

Supplementary Movie S1 


\section{Competing interests}

The authors declare no competing financial interests.

\section{Code availability}

The code used in this paper is available from the corresponding author upon reasonable request.

\section{Data availability}

The data that support the plots within this paper and other findings of this study are available from the corresponding author upon reasonable request. 
